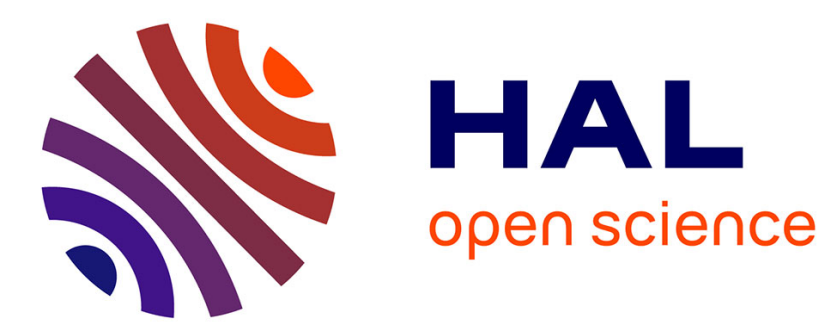

\title{
Inverted pendulum stabilization: Characterization of codimension-three triple zero bifurcation via multiple delayed proportional gains
}

\author{
Islam Boussaada, Irinel-Constantin Morarescu, Silviu-Iulian Niculescu
}

\section{- To cite this version:}

Islam Boussaada, Irinel-Constantin Morarescu, Silviu-Iulian Niculescu. Inverted pendulum stabilization: Characterization of codimension-three triple zero bifurcation via multiple delayed proportional gains. Systems and Control Letters, 2015, 82, pp.1-9. 10.1016/j.sysconle.2015.03.002 hal-01162266

\author{
HAL Id: hal-01162266 \\ https://hal.science/hal-01162266
}

Submitted on 10 Jun 2015

HAL is a multi-disciplinary open access archive for the deposit and dissemination of scientific research documents, whether they are published or not. The documents may come from teaching and research institutions in France or abroad, or from public or private research centers.
L'archive ouverte pluridisciplinaire HAL, est destinée au dépôt et à la diffusion de documents scientifiques de niveau recherche, publiés ou non, émanant des établissements d'enseignement et de recherche français ou étrangers, des laboratoires publics ou privés. 


\title{
Inverted Pendulum Stabilization: Characterization of Codimension-Three Triple Zero Bifurcation Via Multiple Delayed Proportional Gains
}

\author{
Islam Boussaada $^{\mathrm{a}, \mathrm{b}}$, Irinel-Constantin Morărescu ${ }^{\mathrm{c}}$, Silviu-Iulian Niculescu ${ }^{\mathrm{a}}$ \\ ${ }^{a}$ Laboratoire des Signaux et Systèmes, CNRS-Supélec-Université Paris Sud \\ 3 rue Joliot-Curie, 91192 Gif-sur-Yvette cedex (France) \\ ${ }^{b}$ Laboratoire de Modélisation et Calcul Scientifique, IPSA \\ 7 rue Maurice Grandcoing, 94200 Ivry-sur-Seine (France) \\ ${ }^{c}$ Université de Lorraine, CRAN, UMR 7039 and CNRS, CRAN, UMR 7039, \\ 2 avenue de la forêt de Haye, 54516 Vandoeuvre-lès-Nancy Cedex (France)
}

\begin{abstract}
The paper considers the problem of stabilization of systems possessing a multiple zero eigenvalue at the origin. The controller that we propose, uses multiple delayed measurements instead of derivative terms. Doing so, we increase the performances of the closed loop in presence of system uncertainties and/or noisy measurements. The problem formulation and the analysis is presented through a classical engineering problem which is the stabilization of an inverted pendulum on a cart moving horizontally. On one hand, we perform a nonlinear analysis of the center dynamics described by a three dimensional system of ordinary differential equations with a codimension-three triple zero bifurcation. On the other hand, we present the complementary stability analysis of the corresponding linear time invariant system with two delays describing the behavior around the equilibrium. The aim of this analysis is to characterize the possible local bifurcations. Finally, the proposed control scheme is numerically illustrated and discussed.
\end{abstract}

Keywords: Time-Delay, Stability, Delayed Feedback, Nonhyperbolic Dynamics, Center Manifold, Normal Forms, Local Bifurcation Analysis, Inverted Pendulum

\section{Introduction}

In this paper, we employ the classical problem of stabilization of a balancing inverted pendulum on a horizontally moving cart (see for instance $[4,11,19,26,31,32]$ ) to illustrate the control design and performances of delayed proportional controllers. This problem is often used to discuss new ideas in control of nonlinear dynamical systems. This is certainly due to the richness of its dynamics despite the relative simple structure of the physical system. Among possible applications, we emphasize the modeling of the human balance control [22].

Email addresses: islam.boussaada@lss.supelec.fr (Islam Boussaada), constantin.morarescu@univ-lorraine.fr (Irinel-Constantin Morărescu), Silviu.Niculescu@lss.supelec.fr (Silviu-Iulian Niculescu) 
It is well known that the pendulum has two equilibria, one is stable and it corresponds to the pendulum pointing downwards while the other one is unstable and corresponds to the upward position of the pendulum (inverted pendulum). Therefore, the pendulum can be maintained in the upward position only in presence of an appropriate control input. F.M. Atay pointed out (see [4]) that a simple position feedback is not sufficient to obtain satisfactory closed-loop performances. In order to solve the problem one needs additional knowledge such as the rate of change of the position. Thus, a classical controller will contain a derivative feedback term. In [4] the author proposed a proportional minus delay controller (PMD) to obtain asymptotic stability of undamped second-order systems modeling an inverted pendulum. Doing so, the effect of the derivative term is obtained by using a delayed feedback. A proportional controller that locally maintain the pendulum in the upright position was also designed in [19]. In this work it is shown that, when the proportional controller is delayed and the time-delay is not too large, the controller still locally stabilizes the system. Among other results, the authors show the loss of stability when the delay exceeds a critical value, a supercritical Andronov-Hopf Bifurcation [18] occurs generating stable limit cycles.

To the best of the authors' knowledge, PMD controllers were first introduced by I.H. Suh \& Z. Bien in [35] where it is shown that the conventional P-controller equipped with an appropriate time-delay performs an averaged derivative action and thus can replace the PD-controller. It was emphasized that this strategy provides quick responses to input changes but also the insensitiveness to high-frequency noise.

More recently ([32]), J. Sieber and \& B. Krauskopf designed a delayed Proportional Derivative (PD) controller that stabilizes the inverted pendulum on a horizontally moving cart. Moreover, they complement the nonlinear analysis with the local stability analysis of the linearized system around equilibrium. The later characterizes all the possible local bifurcations and is based on the center manifold theory and normal forms, which are known to be powerful tools for the local qualitative study of the dynamics. The study emphasized the existence of a codimensionthree triple zero bifurcation. It is also shown that the stabilization of the inverted pendulum in its upright position cannot be achieved by a delayed PD controller when the delay exceeds some critical value $\tau_{c}$. In [33], the authors investigate some modifications of the delayed PD scheme that allows extending the range of the admissible delay by taking into account the angular acceleration. This design presents the drawback that we need to measure the angular acceleration. An alternative possibility is to introduce an artificial delay in the angular position feedback. It is worth noting that, replacing the derivative with its numerical approximation will not allow to directly apply the results in [32]. Indeed, the behavior of a system (even a linear one) may be different from the behavior of its approximation. In [23], it has been shown that using a polynomial function $\left(1-s \frac{\tau}{n}\right)^{n}$ of arbitrary degree $n$ to approximate an exponential $e^{-s \tau}$ allows finding stabilizing controller gains for the approximated system even when they do not necessarily exist for the original one. Furthermore, introducing a deliberately delay was suggested in [30] to solve the static output feedback sliding mode control problem for a broader class of linear uncertain systems. Indeed, it is shown that the reduced order sliding mode dynamics are stabilized by the introduced artificial delay.

The use of PD controller needs the knowledge of the velocity history but in some circumstances we are only able to have approximate measurements due to technological constraints. In absence of measurements of the derivative, a classical idea is to use an observer to reconstruct the state, but this might degrade the performance to some extent [4] and it is, in general, computationally involved for delay systems. To avoid such degradation and since the position measurement can be easily obtained by sensors, in this paper, we restrict our design to delayed 
proportional gains. The starting idea of our work is a result proposed by W. Michiels \& S-I. Niculescu in [27]. As proven there, a chain of $n$ integrators can be stabilized using $n$ distinct delay blocks, where a delay block is described by two parameters: "gain" and "delay". The interest of considering control laws of the form $\sum_{k=1}^{m} \gamma_{k} y\left(t-\tau_{k}\right)$ lies in the simplicity of the controller as well as in its easy practical implementation. The performances of delayed controllers to overcome the challenge of stabilizing the inverted pendulum are emphasized in the following recent works $[4,32,19]$.

The main contribution of the paper is the analysis of a proportional controller with artificial delays that is able to stabilize the inverted pendulum without the use of derivative measurements. This type of controllers will be called multi-delayed-proportional controllers (MDP) in the sequel. Our analysis agrees with the claim of F.M. Atay [4] but extends it by proving that the knowledge of the delayed derivative gain considered in the delayed PD controller [32] can be replaced by using two delayed position values. We firstly use MDP controllers to reach the configuration of multiple-zero eigenvalue described in [32] and secondly, we identify the appropriate parameter values that stabilize the inverted pendulum avoiding the singularity. It is worth mentioning that, if the presence of the root at the origin is independent of the delay values, its multiplicity depends on the existing relations between the delays and the other parameters of the system. Moreover, such a root at the origin admits a bounded multiplicity [29].

We show that, on the center manifold, the considered MDP controller achieves the same trajectories as the delayed PD considered in [32]. Moreover, we point out that using the proposed MDP we are able to obtain the critical parameter values associated with a triple zero singularity for the delayed PD (see Remark 2). In some sense, this can be seen as a discretization of the feedback state derivative. By the way, such a constructive approach has been adopted in a different context for the controller design developed in $[27,17]$. The stability analysis of the delayed linearized system employs the geometrical interpretation of the corresponding characteristic equation proposed in $[25,14,24]$. An alternative technique for studying the stability of this class of systems is proposed in [34]. For more details on the existing techniques, the reader is referred to [16]. We point out that we are providing stability regions in delay parameter space. Thus, rounding the values due to numerical implementation does not generate troubles as far as the delays are far enough of the crossing curves. This issue is known in the literature as fragility of the controller and it mainly appears when the design is made for a continuous system but the implementation is done in a digital fashion. A non-fragile controller allows rounding numerical values without loosing stability properties. A methodology to design non-fragile PI, PD or PID controllers has been presented in previous works of the authors (see for instance [20]). The main idea is to chose the controller parameters that maximize the distance to the closest tangent to the crossing stability manifold (i.e. the manifold that separates to regions with different number of unstable roots).

The remaining part of our paper is organized as follows. First, the model of the inverted pendulum on a cart is introduced as well as some mathematical notions used in the analysis. Next, a double delay block control strategy is presented and analyzed. The analysis of the proposed controller includes the linear stability analysis pointing out the Andronov-Hopf bifurcation as well as the multiple-zero singularity, which suggests a central dynamics analysis. Conclusions, comparisons and future work end the paper. 


\section{Settings and useful notions}

\subsection{Friction free model of an Inverted Pendulum on a Cart}

In the sequel we consider the friction free model presented in [32] by adopting the same notations. Denote the mass of the cart $M$, the mass of the pendulum $m$ and let the relative mass be $\epsilon=m /(m+M)$.

In the dimensionless form, if frictions are neglected, the dynamics of the inverted pendulum on a cart in figure 1 is governed by the following ODE, see also [33]:

$$
\left(1-\frac{3 \epsilon}{4} \cos ^{2}(\theta)\right) \ddot{\theta}+\frac{3 \epsilon}{8} \dot{\theta}^{2} \sin (2 \theta)-\sin (\theta)+D \cos (\theta)=0,
$$

where $D$ represents the horizontal driving force exerted by the control law.

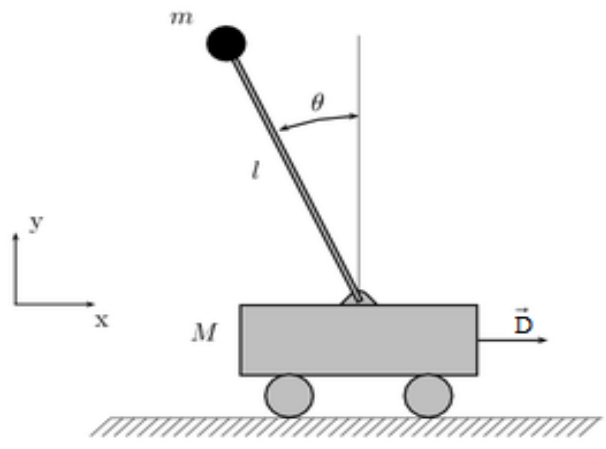

Figure 1: Inverted Pendulum on a cart

In the next section, the horizontal control force will be referred to as position feedback. This was suggested in $[27,17]$ in the context of stabilizing a finite dimensional system consisting of a chain of 2- integrators: $D=\sum_{k=1}^{2} a_{k} \theta\left(t-\tau_{k}\right)$.

In the sequel, we explicitly design the controller that avoids the triple zero singularity.

\subsection{Prerequisites: Space decomposition for time-delay systems}

Consider the general discrete delayed autonomous first-order nonlinear system where its linear and nonlinear quantities are separated as follows:

$$
\frac{d}{d t} x(t)=\sum_{k=0}^{n} A_{k} x\left(t-\tau_{k}\right)+\mathcal{F}\left(x(t), \ldots, x\left(t-\tau_{n}\right)\right),
$$

where $A_{i}$ are $n \times n$ real valued matrices, the delays $\tau_{k}$ are ordered such that $\tau_{j}<\tau_{j}$ when $i<j$, $\tau_{n}=r$ and $\tau_{i} \geq 0$.

The latter system can be written as:

$$
\frac{d}{d t} x=\underset{4}{\mathcal{L} x_{t}+\mathcal{F}\left(x_{t}\right),}
$$


where $x_{t} \in C_{r, n}=C\left([-r, 0], \mathbb{R}^{n}\right), x_{t}(\theta)=x(t+\theta)$ denotes the system state, $\mathcal{L}$ is a bounded linear operator such that $\mathcal{L} \phi=\sum_{k=0}^{n} A_{k} \phi\left(-\tau_{k}\right)$ and $\mathcal{F}$ is assumed to be a sufficiently smooth function mapping $C_{r, n}$ into $\mathbb{R}^{n}$ with $\mathcal{F}(0)=\mathcal{D F}(0)=0$ where $\mathcal{D}$ is the Fréchet derivative. The linear operator $\mathcal{L}$ can be written in the integral form as $\mathcal{L} \phi=\int_{-r}^{0} d \eta(\theta) \phi(\theta)$ where $\eta$ is a real valued $n \times n$ matrix.

The linearization of (3) is given by

$$
\frac{d}{d t} x=\mathcal{L} x_{t}
$$

the solution of which is given by the operator $\mathcal{T}(t)$ defined by $\mathcal{T}(t)(\phi)=x_{t}(., \phi)$ such that $x_{t}(., \phi)(\theta)=x(t+\theta, \phi)$ for $\theta \in[-r, 0]$. This is a strongly continuous semigroup, the infinitesimal generator of which is $\mathcal{A}=\frac{d \phi}{d \theta}$ with the domain

$$
\mathcal{D o m}(\mathcal{A})=\left\{\phi \in C_{r, n}: \frac{d \phi}{d \theta} \in C_{r, n}, \frac{d \phi}{d \theta}=\mathcal{L} \phi\right\} .
$$

It is also known that the spectrum of $\mathcal{A}$ is $\sigma(\mathcal{A})=\sigma_{p}(\mathcal{A})$ (point spectrum) and consists of complex values $\lambda \in \mathbb{C}$ satisfying the characteristic equation $p(\lambda)=0$, (see [21] for further details).

In the spirit of [8], let us denote by $\mathcal{M}_{\lambda}$ the eigenspace associated with $\lambda \in \sigma(\mathcal{A})$. We define $C_{r, n}^{*}=C\left([-r, 0], \mathbb{R}^{n *}\right)$ where $\mathbb{R}^{n *}$ is the space of $\mathrm{n}$-dimensional row vectors and consider the bilinear form on $C_{r, n}^{*} \times C_{r, n}$ as proposed in [15]:

$$
(\psi, \phi)=\phi(0) \psi(0)+\int_{-r}^{0} \int_{0}^{\theta} \psi(\tau-\theta) d \eta(\theta) \phi(\tau) d \tau .
$$

Let $\mathcal{A}^{T}$ be the transposed operator of $\mathcal{A}$, i.e., $(\psi, \mathcal{A} \phi)=\left(\mathcal{A}^{T} \psi, \phi\right)$. The following result enables the decomposition of the space $C_{r, n}$.

Theorem 2.1 (Banach space decomposition, [15]). Let $\Lambda$ be a nonempty finite set of eigenvalues of $\mathcal{A}$, let $P=\operatorname{span}\left\{\mathcal{M}_{\lambda}(\mathcal{A}), \lambda \in \Lambda\right\}$ and $P^{T}=\operatorname{span}\left\{\mathcal{M}_{\lambda}\left(\mathcal{A}^{T}\right), \lambda \in \Lambda\right\}$. Then $P$ is invariant under $\mathcal{T}(t), t \geq 0$ and there exists a space $Q$, also invariant under $\mathcal{T}(t)$, such that $C_{r, n}=P \bigoplus Q$. Furthermore, if $\Phi=\left(\phi_{1}, \ldots, \phi_{m}\right)$ is a basis of $P$, and $\Psi=\operatorname{col}\left(\psi_{1}, \ldots, \psi_{m}\right)$ is a basis of $P^{T}$ in $C_{r, n}^{*}$ such that $(\Phi, \Psi)=I d$, then

$$
\begin{aligned}
& Q=\left\{\phi \in C_{r, n} \backslash(\Psi, \phi)=0\right\} \text { and } \\
& P=\left\{\phi \in C_{r, n} \backslash \exists b \in \mathbb{R}^{m}: \phi=\Phi b\right\} .
\end{aligned}
$$

Also, $\mathcal{T}(t) \Phi=\Phi e^{B t}$, where $B$ is an $m \times m$ matrix such that $\sigma(B)=\Lambda$.

Consider the extension of the space $C_{r, n}$ that contains continuous functions on $[-r, 0)$ with a possible jump discontinuity at 0 , we denote this space $B C$. A given function $\xi \in B C$ can be written as $\xi=\varphi+X_{0} \alpha$, where $\varphi \in C_{r, n}, \alpha \in \mathbb{R}^{n}$ and $X_{0}$ is defined by $X_{0}(\theta)=0$ for $-r \leq \theta<0$ and $X_{0}(0)=I d_{n \times n}$. Then the Hale-Verduyn Lunel bilinear form [15] can be extended to the space $C_{r, n}^{*} \times B C$ by $\left(\psi, X_{0}\right)=\psi(0)$ and the infinitesimal generator $\mathcal{A}$ extends to an operator $\tilde{\mathcal{A}}$ (defined in $C^{1}$ ) onto the space $B C$ as follows:

$$
\tilde{\mathcal{A}} \phi=\mathcal{A} \phi+X_{0}\left[\mathcal{L} \phi-\phi^{\prime}\right]
$$


Under the above consideration one can write equation (3) as an abstract ODE:

$$
\dot{x}_{t}=\tilde{\mathcal{A}} x_{t}+X_{0} \mathcal{F}\left(x_{t}\right) .
$$

Due to the projection $\Pi: B C \rightarrow P$ defined by $\Pi\left(\varphi+X_{0} \alpha\right)=\Phi[(\Psi, \varphi)+\Psi(0) \alpha]$ and the state decomposition such that $x_{t}=\Phi y(t)+z_{t}$ where $y(t) \in \mathbb{R}^{m}$ and $z_{t} \in Q$. Then, the equation (3) can be split into two equations. Our interest lies essentially in the evolution equation for the finite dimensional part of the space, i.e., the first equation of the following system:

$$
\left\{\begin{array}{l}
\dot{y}=B y+\Psi(0) \mathcal{F}(\Phi y+z), \\
\dot{z}=\tilde{A}_{Q} z+(I-\pi) \mathcal{F}(\Phi y+z) .
\end{array}\right.
$$

For more details and insights, see for instance, $[15,12]$. Assume now that $\mathcal{F}$ depends on some parameter $p$, and denote the semiflow generated by $(8)$ as $\mathcal{S}(t, y, z, p)$, then $\mathcal{S}$ is equivalent to the semiflow generated by (3):

Theorem 2.2 (Existence and Properties of the Center Manifold). Let $k>0$ and $\mathcal{U}_{y} \times \mathcal{U}_{z} \times \mathcal{U}_{p}$ be a small neighborhood of $\left(0,0, p_{0}\right) \in \mathbb{R}^{n} \times Q \times \mathbb{R}^{m}$. There exists a graph $\omega: \mathcal{U}_{y} \times \mathcal{U}_{p} \rightarrow Q$ of smoothness $C^{k}$ such that the following statements hold.

1. (Invariance) The manifold $\left\{(y, z) \in \mathcal{U}_{y} \times Q: z=\omega(y, p)\right\}$ is invariant with respect to $\mathcal{S}$ relative to $\mathcal{U}_{y} \times \mathcal{U}_{z}$.

2. (Exponential attraction) Let $(y, z)$ be such that $\mathcal{S}(y, z, p) \in \mathcal{U}_{y} \times \mathcal{U}_{z} \forall t \geq 0$. Then there exists $\tilde{y}$ and $\tilde{t} \geq 0$ such that $\|\mathcal{S}(t+\tilde{t}, y, z, p)-\mathcal{S}(t, \tilde{y}, \omega(\tilde{y}), p)\| \leq K e^{\frac{-t_{0}}{2}}$ for all $t>0$.

\subsection{Multiplicity of the root at the origin: Polya-Szegö Bound}

Consider the quasipolynomial function $\Delta: \mathbb{C} \times \mathbb{R}^{N} \rightarrow \mathbb{C}$ of the form:

$$
\Delta(\lambda, \tau)=P_{0}(\lambda)+\sum_{k=1}^{N} P_{k}(\lambda) e^{-\tau_{k} \lambda},
$$

where $\tau_{k}, k=1, \ldots, N$ are constant delays such that $\tau_{1}<\tau_{2} \ldots<\tau_{N}$ and $\tau=\left(\tau_{1}, \ldots, \tau_{N}\right)$ is the delays vector. Without any loss of generality, assume that the polynomial $P_{0}$ is a monic of degree $n$ in $\lambda$ and the polynomials $P_{k}$ are such that $\operatorname{deg}\left(P_{k}\right) \leq n-1, \forall 1 \leq k \leq N$. One can prove that the quasipolynomial function (9) admits an infinite number of zeros, see for instance $[1,5]$. However, the multiplicity of any root is bounded, in particular the root at the origin. The following result, due to Polya-Szegö, gives to such a bound:

Proposition 2.3 (Pólya-Szegö, [29], pp.144). Let $\tau_{1}, \ldots, \tau_{N}$ denote real numbers such that $\tau_{1}<$ $\tau_{2}<\ldots<\tau_{N}$ and $d_{1}, \ldots, d_{N}$ positive integers such that $d_{1}+d_{2}+\ldots+d_{N}=D$.

Let $f_{i, j}(s)$ stand for the function $f_{i, j}(s)=s^{i-1} e^{\tau_{j} s}$, for $1 \leq i \leq d_{j}$ and $1 \leq j \leq N$. Let $\sharp$ be the number of zeros of the function

$$
f(s)=\sum_{1 \leq j \leq N, 1 \leq i \leq d_{j}} c_{i, j} f_{i, j}(s),
$$

that are contained in the horizontal strip $\alpha \leq \mathcal{I}(z) \leq \beta$.

Assuming that

$$
\sum_{1 \leq k \leq d_{1}}\left|c_{k, 1}\right|>0 \text { and } \sum_{1 \leq k \leq d_{N}}\left|c_{k, N}\right|>0 \text {, }
$$


then

$$
\frac{\left(\tau_{N}-\tau_{1}\right)(\beta-\alpha)}{2 \pi}-D+1 \leq \sharp \leq \frac{\left(\tau_{N}-\tau_{1}\right)(\beta-\alpha)}{2 \pi}+D-1 .
$$

The proof of the mentioned Pólya-Szegö result is mainly based on Rouché's Lemma [1]. It can be generically exploited to establish a bound for the multiplicity of the zero spectral value that we denote by $\sharp_{P S}$. Indeed, setting $\alpha=\beta=0$ we get $\sharp_{P S} \leq D-1$. This gives a sharp bound when all the system parameters are left free. Nevertheless, it is obvious that the PólyaSzegö bound does not change if certain coefficients $c_{i, j}$ vanish without affecting the degree of the quasipolynomial function.

The above result from [6] sets the necessary and sufficient conditions guaranteeing the PólyaSzegö multiplicity for the zero singularity.

Proposition 2.4 ([6]). The multiplicity of the zero singularity reaches the Pólya-Szegö a bound if and only if the parameters of (9) satisfy simultaneously:

$$
a_{0, k}=-\sum_{i=1}^{N}\left[a_{i, k}+\sum_{j=0}^{k-1} \frac{(-1)^{j+1} a_{i, j} \tau_{i}^{k-j}}{(k-j) !}\right], \quad 0 \leq k \leq \#-1 .
$$

where $a_{i, l}$ stands for the coefficient of the monomial $\lambda^{l}$ for the polynomial $P_{i}$ for $1 \leq i \leq N$.

Furthermore, it is shown in [6] that, under the nondegeneracy of an appropriate Birkhoff matrix, the multiplicity of the zero root for the quasipolynomial function (9) cannot be larger than $n$ plus the number of nonzero coefficients of the polynomial family $\left(P_{k}\right)_{1 \leq k \leq N}$, which is sharper than $\sharp_{P S}$.

Remark 1. Increasing the multiplicity of the zero singularity induces richer (more complex) dynamics in the neighborhood of the steady state. We show in the sequel that the proposed methodology is able to stabilize the solutions around the unstable equilibrium point even when the multiplicity of the zero singularity reaches its optimal value.

\section{Double Delay Block}

Let the horizontal driving force exerted by the control law be $D=a \theta\left(t-\tau_{1}\right)+b \theta\left(t-\tau_{2}\right)$. Thus, equation (1) can be written as a Delay-Differential Equation (DDE) of the form:

$$
\dot{x}=f\left(x(t), x\left(t-\tau_{1}\right), x\left(t-\tau_{2}\right), \lambda\right),
$$

where $x=\left(x_{1}, x_{2}\right)^{T}=(\theta(t), \dot{\theta}(t))^{T}$ and $\lambda=\left(a, b, \tau_{1}, \tau_{2}\right)$. The right hand side $f: \mathbb{R}^{2} \times \mathbb{R}^{2} \times \mathbb{R}^{2} \times$ $\mathbb{R}^{4} \rightarrow \mathbb{R}^{2}$ is given by:

$$
\begin{aligned}
& f_{1}(x, y, z, \lambda)=x_{2} \\
& f_{2}(x, y, z, \lambda)=\frac{-\frac{3 \epsilon}{8} \sin \left(2 x_{1}\right) x_{2}^{2}+\sin \left(x_{1}\right)-\cos \left(x_{1}\right)\left(a y_{1}+b z_{1}\right)}{1-\frac{3 \epsilon}{4} \cos ^{2}\left(x_{1}\right)} .
\end{aligned}
$$

where $y=\left(\theta\left(t-\tau_{1}\right), \dot{\theta}\left(t-\tau_{1}\right)\right)^{\top}$ and $z=\left(\theta\left(t-\tau_{2}\right), \dot{\theta}\left(t-\tau_{2}\right)\right)^{\top}$.

The phase space of (11)-(12) is the space of continuous functions over the delay interval $\left[-\max \left(\tau_{1}, \tau_{2}\right), 0\right]$ with values in $\mathbb{R}^{2}$. Obviously $f(-x,-y,-z, \lambda)=-f(x, y, z, \lambda)$, and thus, the origin represents always an equilibrium point. Furthermore, any solution of (11)-(12) is either symmetric or skew-symmetric with respect to the origin. 


\subsection{Linear Stability Analysis}

As emphasized in Remark 1, we consider the optimal multiplicity for the zero spectral value (the most complex configuration). Taking the relative mass $\epsilon=\frac{3}{4}$ guaranties such an optimal multiplicity. Note that, if $\epsilon \in(0,1)$, and regardless of the multiplicity of the zero singularity, all the following steps apply albeit in a simpler fashion. One easily checks that the zero multiplicity is less than four (otherwise $\tau_{1}$ and $\tau_{2}$ have opposite signs). Indeed, the linearization of $f$ with respect to its three arguments, $x, y$ and $z$ at the origin is given by:

$$
\begin{aligned}
& \partial_{1} f(0,0,0, \lambda)=\left[\begin{array}{cc}
0 & 1 \\
\frac{16}{7} & 0
\end{array}\right], \partial_{2} f(0,0,0, \lambda)=\left[\begin{array}{cc}
0 & 0 \\
-\frac{16}{7} a & 0
\end{array}\right], \\
& \partial_{3} f(0,0,0, \lambda)=\left[\begin{array}{cc}
0 & 0 \\
-\frac{16}{7} b & 0
\end{array}\right] .
\end{aligned}
$$

Then, the characteristic function reads:

$$
\Delta(z)=z^{2}+\frac{16}{7}\left(a \mathrm{e}^{-z \tau_{1}}+b \mathrm{e}^{-z \tau_{2}}-1\right) .
$$

Several approaches can be used for characterizing imaginary crossing roots of quasipolynomials as well as their crossing directions, see for instance [28, 34]. Here, we follow the idea proposed by $[14,25]$. We introduce the stability crossing curves $\mathbf{T}$, which represents the set of $\left(\tau_{1}, \tau_{2}\right)$ such that $\Delta(z)$ has imaginary solutions. As the parameters $\left(\tau_{1}, \tau_{2}\right)$ cross the stability crossing curves, some characteristic roots cross the imaginary axis. Introduce also the crossing set $\Omega$, which is defined as the collection of all $\omega>0$ such that there exists a parameter pair $\left(\tau_{1}, \tau_{2}\right)$ such that $\Delta\left(j \omega, \tau_{1}, \tau_{2}\right)=0$. Using Proposition 3.1 in [14] the following stability characterization can be deduced:

Proposition 3.1. For $a+b<1$, the crossing set $\Omega$ is empty so the system is delay independently unstable. When $a+b>1$, the crossing set $\Omega$ reduces to one interval $\left(0, \omega^{r}\right]$ and $\mathbf{T}$ is a series of open-ended curves $\mathbf{T}_{u, v}^{ \pm}$where $\mathbf{T}_{u, v}^{-}$and $\mathbf{T}_{u, v+1}^{+}$are connected at $\omega^{r}$.

We emphasize that

- when $a+b>1, a>b$ the crossing set $\Omega$ contains only simple solution of $\Delta$

- when $a+b>1, a<b$ one has frequencies $\omega \in \Omega$ which are solution of multiplicity 2 for $\Delta$.

The function $\Delta$ has a root 0 along the red solid red curve in figure 4 given by $a+b=1$, where the origin undergoes a pitchfork bifurcation.

In a similar way, we can introduce the stability crossing curves $\mathbf{A}$ in the parameter space $(a, b)$ and the corresponding crossing set $\Gamma$. Thus $\mathbf{A}$ is the set of $(a, b)$ for which $\Delta(z)$ has imaginary solutions while $\Gamma$ consist of those frequencies $\omega$ for which there exists a parameter pair $(a, b)$ such that $\Delta(j \omega, a, b)=0$. The stability analysis in the $(a, b)$ parameter space is summarized as follows:

Proposition 3.2. The crossing set $\Gamma$ consists of all frequencies satisfying:

$$
0<\omega<\left|\frac{\pi}{\tau_{1}-\tau_{2}}\right|,
$$



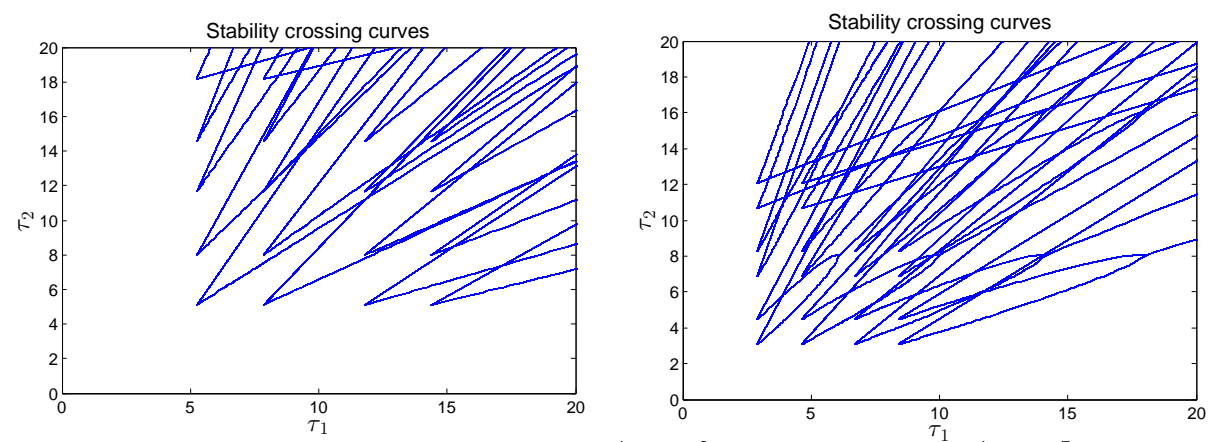

Figure 2: Stability in delay parameter space for $a=\frac{4}{5}, b=\frac{3}{5}$ on the left and for $a=\frac{4}{5}, b=\frac{7}{5}$ on the right

and the crossing curves are defined by:

$$
\left\{\begin{array}{l}
a=-\frac{\sin \left(\omega \tau_{2}\right)}{\sin \left(\omega \tau_{1}\right)} b, \\
b=\frac{1+\frac{7}{16} \omega^{2}}{\cos \left(\omega \tau_{2}\right)-\frac{\sin \left(\omega \tau_{2}\right)}{\sin \left(\omega \tau_{1}\right)} \cos \left(\omega \tau_{1}\right)}
\end{array} \quad \forall \omega \in \Gamma .\right.
$$
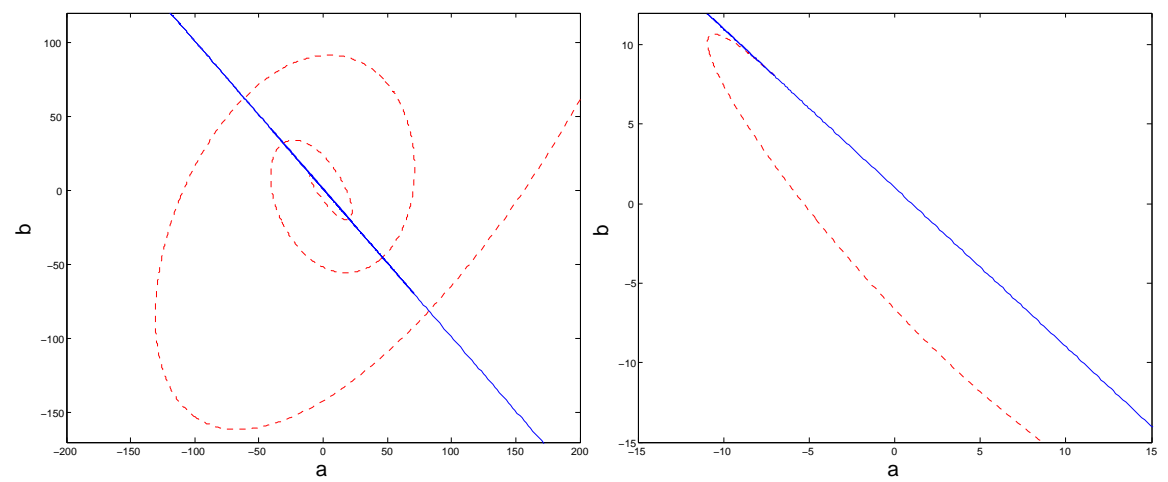

Figure 3: Left: Dashed curve represents the stability crossing curve in $(a, b)$ parameter space for $\tau_{1}=1, \tau_{2}=\frac{7}{8}$ while the solid curve is the line $a+b=1$. Right: Zoom in the neighborhood of $\omega=0$.

It is always possible to normalize one of the delays by a simple scaling of time. Without any lack of generality, assume that $\tau_{1}=1$. As can be seen in figure 3, when $\omega$ approaches 0 the crossing curve approaches the line $a+b=1$.

The quasipolynomial function $\Delta$ has a purely imaginary root $i w$ if the gains $a$ and $b$ satisfy (13). Thus, equation (13) defines the curve of Hopf Bifurcation in the $(a, b)$ plane, dashed blue line in figure 4 . We note also that substituting $w=0$ in the expressions of $a$ and $b$ allows deriving the values of the gain guarateeing an eigenvalue at zero of algebraic multiplicity 2 . Substituting these values into the third derivative of characteristic function $\Delta$ and replacing $\tau_{2}=\tau_{1}+\delta$ leads to the control loop latency $\tau_{1}^{*}=\frac{1}{2}\left(\sqrt{\delta^{2}+8-6 \epsilon}-\delta\right)$ already identified in [33] where the linear 

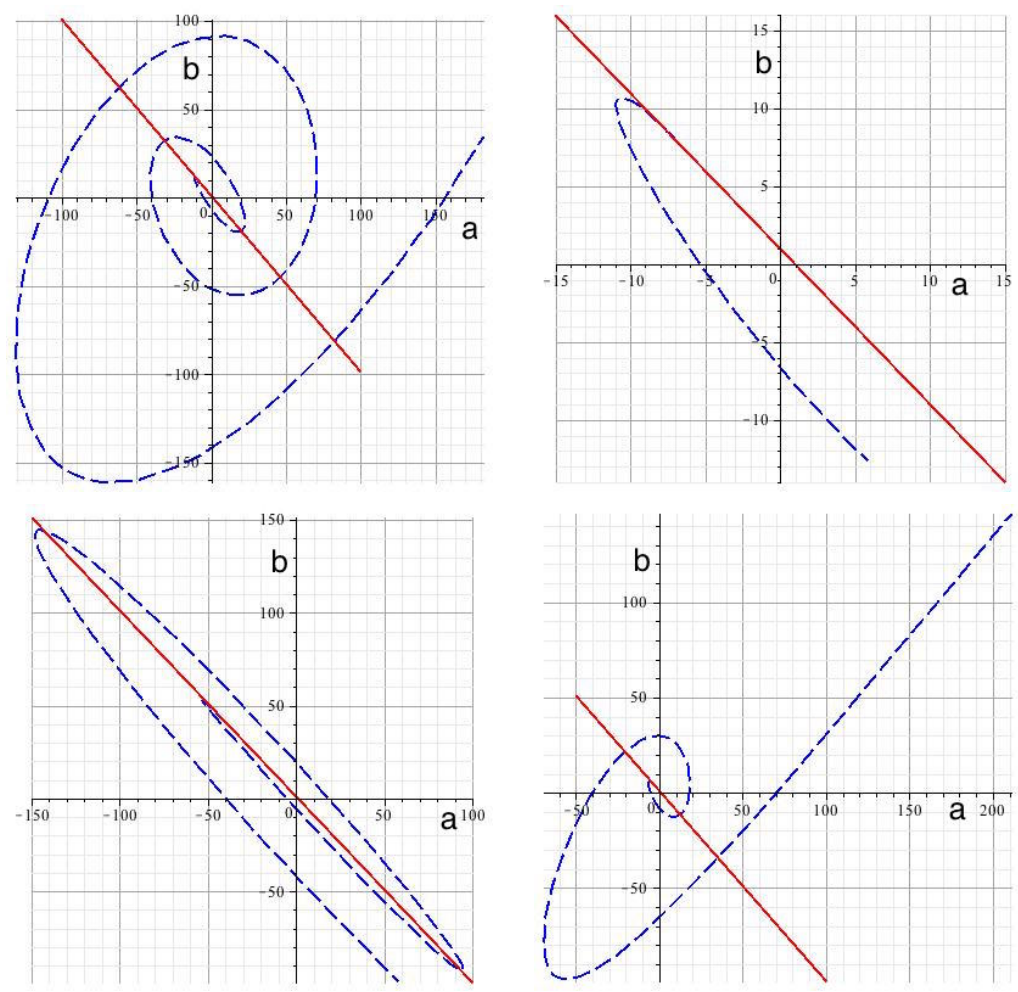

Figure 4: Bifurcations curves of (11)-(12) in the gains $(\mathrm{a}, \mathrm{b})$ plan (solid red=Pitchfork, discontinuous blue=Hopf) with $\tau_{1}=1$ and $\tau_{2}$ such that (top left) $\tau_{2}=\frac{7}{8}$ (top right) $\tau_{2}=\frac{7}{8}$ the neighborhood of $(-7,8)$ (bottom left) $\tau_{2}=\frac{7}{8}+\frac{1}{10}$ (bottom right) $\tau_{2}=\frac{7}{8}-\frac{1}{10}$

analysis and a comparative study is made (PMD vs Acceleration-dependent control). It is also shown that the "optimal" value of the control loop latency is reached when $\delta=0$.

Remark 2. In [32], the authors consider $D=a \theta(t-\tau)+b \dot{\theta}(t-\tau)$ and prove that the truncated cubic central dynamics reduces to:

$$
\dot{u}=\left[\begin{array}{ccc}
0 & 1 & 0 \\
0 & 0 & 1 \\
\alpha & \beta & \gamma
\end{array}\right] u+\left[\begin{array}{c}
0 \\
0 \\
u_{1}^{3}
\end{array}\right],
$$

where $\alpha, \beta$ and $\gamma$ are small parameters, showing that the triple zero singularity can be avoided.

To recover the analysis established in [32], consider a horizontal driving force $D=a \theta(t-$ $\left.\tau_{1}\right)+\tilde{b}\left(\theta\left(t-\tau_{1}\right)-\theta\left(t-\tau_{1}-h\right)\right)$ where $\tilde{b}=\frac{b}{h}$ and, without loss of generality, assume that $\tau_{1} \leq \tau_{2}$, that is $h \geq 0$. Then $\lim _{h \rightarrow 0} D=a \theta\left(t-\tau_{1}\right)+b \dot{\theta}\left(t-\tau_{1}\right)$. Thus, the configuration of a triple zero eigenvalue is ensured by the set of conditions $\left\{a=1, b=\tau_{1}, h=-\frac{-1+\tau_{1}^{2}}{\tau_{1}}, \tau_{2}=\tau_{1}^{-1}\right\}$. By imposing $h$ to converge to zero, $\tau_{1}$ tends to $\tau_{1}^{*}=1$ so that the gain $b$ tends to $b^{*}=\tau_{1}^{*}=1$ which are, as expected, the identified values in [32]. In Figure 5 we represent the delay difference $\tau_{2}-\tau_{1}$. 


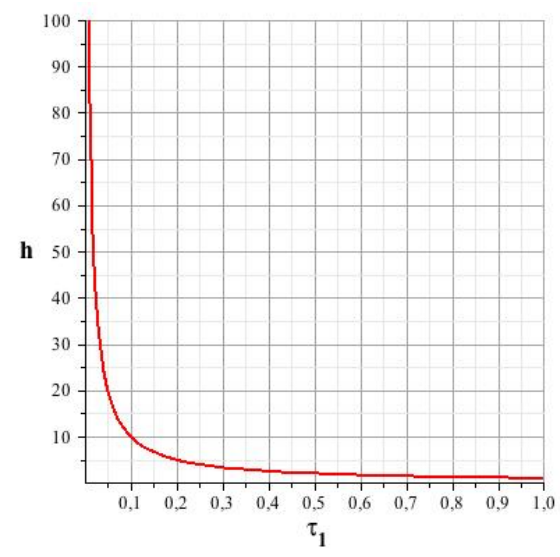

Figure 5: The delay difference $h=\tau_{2}-\tau_{1}$ vs $\tau_{1}$ for conserving a triple eigenvalue at zero

Remark 3. It is worth noting that the delay normalization (setting $\tau_{1}=1$ ) does not affect the existence of the triple zero eigenvalue. Indeed, when $\tau_{1}$ is left free the set of conditions:

$$
\left\{a=\frac{-7}{8 \tau_{1}-7}, b=\frac{8 \tau_{1}^{2}}{8 \tau_{1}^{2}-7}, \tau_{2}=\frac{7}{8 \tau_{1}}\right\}
$$

ensures this configuration.

Now, to argue the above normalization, let us consider the simplest demonstrative example; a scalar equation with two delays: $\dot{x}=a_{0} x(t)+a_{1} x\left(t-\tau_{1}\right)+a_{2} x\left(t-\tau_{2}\right)$. We introduce the following time scaling $t=\zeta \tau_{1}$ and consider a new variable $v(\zeta)=x(t)$. Thus, the dynamic of the new variable $v$ is governed by

$$
\begin{aligned}
v^{\prime}(\zeta)=\frac{d v(\zeta)}{d \zeta}=\dot{x}(t) \frac{d t}{d \zeta} & =\tau_{1}\left(a_{0} x(t)+a_{1} x\left(t-\tau_{1}\right)+a_{2} x\left(t-\tau_{2}\right)\right) \\
& =b_{0} v(\zeta)+b_{1} v(\zeta-1)+b_{2} v(\zeta-\tau)
\end{aligned}
$$

where $b_{i}=a_{i} / \tau_{1}$ for $i=0, \ldots, 2$ and $\tau=\tau_{2} / \tau_{1}$. Which justifies as expected the adopted normalization.

\subsection{Normal Form of the Central Dynamics}

Several approaches exist to establish the decomposition of the Banach space of continuous functions, see for instance [7, 32, 2]. In the sequel, we follow the elegant approach based on the computation of the spectral projection presented in [32]. It is worth to mention that this spectral projection is mainly based on the bilinear form presented in the previous section justifying the universality of the spectral decomposition modulo the chosen base of the generalized eigenspace associated with pure imaginary spectral values.

The parameter point $\lambda_{0}=\left(a_{0}, b_{0}, \tau_{1}{ }^{*}, \tau_{2}{ }^{*}\right)=\left(-7,8,1, \frac{7}{8}\right)$ characterizes a triple zero eigenvalue at the origin. As said above, it is always possible to re-scale the time in order to normalize one of the delays to 1 ( $\tau_{1}$ becomes 1 and $\tau_{2}$ becomes $\tau_{2} / \tau_{1}$ ) so the rescaled system (11)-(12) 
reads

$$
\left\{\begin{array}{l}
f_{1}(., \lambda)=x_{2}, \\
f_{2}(., \lambda)=\frac{\left(-\frac{9}{32} \sin \left(2 x_{1}\right) x_{2}^{2}+\tau_{1}^{2} \sin \left(x_{1}\right)-\tau_{1}^{2} \cos \left(x_{1}\right)\left(a y_{1}+b z_{1}\right)\right)}{1-\frac{9}{16}\left(\cos \left(x_{1}\right)\right)^{2}} .
\end{array}\right.
$$

Let $X$ be the Banach space $\mathbb{R}^{2} \times C\left([-1,0], \mathbb{R}^{2}\right)$. Consider

$$
D(H):=\left\{(y, \tilde{y}) \in \mathbb{R}^{2} \times C^{1}\left([-1,0], \mathbb{R}^{2}\right): \tilde{y}(0)=y\right\} \subset X,
$$

and define the linear operator

$$
\begin{aligned}
& H: D(H) \subset X \rightarrow X, \\
& H\left[\begin{array}{c}
y \\
\tilde{y}
\end{array}\right]=\left[\begin{array}{c}
\partial_{1} f(0,0,0, \lambda) \tilde{y}(0)+\partial_{2} f(0,0,0, \lambda) \tilde{y}(-1)+\partial_{3} f(0,0,0, \lambda) \tilde{y}\left(-\frac{7}{8}\right) \\
\partial_{s} \tilde{y}
\end{array}\right],
\end{aligned}
$$

where the spatial variable in $C^{1}\left([-1,0], \mathbb{R}^{2}\right)$ is denoted by $s$. The operator $H$ is a closed unbounded operator. It generates a strongly continuous semigroup $T(t)$ of bounded operators in $Y=\left\{(y, \tilde{y}) \in \mathbb{R}^{2} \times C\left([-1,0], \mathbb{R}^{2}\right): \tilde{y}(0)=y\right\} \subset X$. The semigroup $T($.) is compact for $t>1$.

Let $g$ be the nonlinear part of $f$ i.e.

$$
g\left(\left[\begin{array}{l}
y \\
\tilde{y}
\end{array}\right], \lambda\right)=\left[\begin{array}{c}
g_{0}\left(\tilde{y}(0), \tilde{y}(-1), \tilde{y}\left(-\frac{7}{8}\right), \lambda\right) \\
0
\end{array}\right],
$$

where

$$
\begin{aligned}
& g_{0}\left(\tilde{y}(0), \tilde{y}(-1), \tilde{y}\left(-\frac{7}{8}\right), \lambda\right)=f\left(\tilde{y}(0), \tilde{y}(-1), \tilde{y}\left(-\frac{7}{8}\right), \lambda\right)- \\
& \left(\partial_{1} f(0,0,0, \lambda) \tilde{y}(0)+\partial_{2} f(0,0,0, \lambda) \tilde{y}(-1)+\partial_{3} f(0,0,0, \lambda) \tilde{y}\left(-\frac{7}{8}\right)\right) .
\end{aligned}
$$

System (11)-(12) is equivalent to the autonomous evolution equation:

$$
\dot{x}=H x+g(x, \lambda) .
$$

The decomposition of the Banach space reads $X=P \bigoplus Q$ where $P$ is the $H$-invariant generalized eigenspace associated to the triple zero singularity and is isomorphic to $\mathbb{R}^{3}$, and $Q$ is also $H$ invariant of infinite dimension. Next, we compute $\Phi$ a basis of $P$ satisfying $H \Phi=\Phi J$, where:

$$
\Phi(s)=\left[\phi_{1}, \phi_{2}, \phi_{3}\right]=\left[\begin{array}{ccc}
1 & 0 & 1 \\
0 & 1 & 0 \\
1 & s & \frac{s^{2}}{2}+1 \\
0 & 1 & s
\end{array}\right] \text {, and } J=\left[\begin{array}{ccc}
0 & 1 & 0 \\
0 & 0 & 1 \\
0 & 0 & 0
\end{array}\right] \text {. }
$$

We also compute the invariant spectral projection $\mathcal{P}: X \rightarrow P$ satisfying $\mathcal{P}_{x}=\operatorname{Res}_{z=0}(z I-H)^{-1}$. Thus, $\mathcal{P}_{x}=l_{1}(x) \phi_{1}+l_{2}(x) \phi_{2}+l_{3}(x) \phi_{3}$ where: 


$$
\begin{aligned}
l_{1}(x) & =\frac{169}{300} \tilde{y}_{1}(0)-\frac{222179}{144000} \tilde{y}_{2}(0)-\frac{222179}{9000} \int_{0}^{1} \tilde{y}_{1}(t-1) d t \\
+ & \frac{222179}{7875} \int_{0}^{\frac{7}{8}} \tilde{y}_{1}\left(t-\frac{7}{8}\right) d t-\frac{676}{75} \int_{0}^{1} t \tilde{y}_{1}(t-1) d t+\frac{5408}{525} \int_{0}^{\frac{7}{8}} t \tilde{y}_{1}\left(t-\frac{7}{8}\right) d t \\
& +\frac{64}{5} \int_{0}^{1} t^{2} \tilde{y}_{1}(t-1) d t-\frac{512}{35} \int_{0}^{\frac{7}{8}} t^{2} \tilde{y}_{1}\left(t-\frac{7}{8}\right) d t, \\
l_{2}(x) & =\frac{8}{5} \tilde{y}_{1}(0)+\frac{169}{300} \tilde{y}_{2}(0)+\frac{676}{75} \int_{0}^{1} \tilde{y}_{1}(t-1) d t-\frac{5408}{525} \int_{0}^{\frac{7}{8}} \tilde{y}_{1}\left(t-\frac{7}{8}\right) d t \\
& -\frac{128}{5} \int_{0}^{1} t \tilde{y}_{1}(t-1) d t+\frac{1024}{35} \int_{0}^{\frac{7}{8}} t \tilde{y}_{1}\left(t-\frac{7}{8}\right) d t, \\
l_{3}(x) & =\frac{8}{5} \tilde{y}_{2}(0)+\frac{128}{5} \int_{0}^{1} \tilde{y}_{1}(t-1) d t-\frac{1024}{35} \int_{0}^{\frac{7}{8}} \tilde{y}_{1}\left(t-\frac{7}{8}\right) d t,
\end{aligned}
$$

which allows decomposing (17) to:

$$
\begin{aligned}
\dot{v} & =J v+\Psi(0) g_{0}\left(\tilde{\Phi}(0) v+\tilde{w}_{0}, \tilde{\Phi}(-1) v+\tilde{w}(-1), \tilde{\Phi}\left(-\frac{7}{8}\right) v+\tilde{w}\left(-\frac{7}{8}\right)\right) \\
\dot{\tilde{w}}_{0} & =\partial_{1} f \tilde{w}_{0}+\partial_{2} f \tilde{w}(-1)+\partial_{3} f \tilde{w}\left(\frac{7}{8}\right) \\
& +(I-\Phi(0) \Psi(0)) g_{0}\left(\tilde{\Phi}(0) v+\tilde{w}_{0}, \tilde{\Phi}(-1) v+\tilde{w}(-1), \tilde{\Phi}\left(-\frac{7}{8}\right) v+\tilde{w}\left(-\frac{7}{8}\right)\right) \\
\dot{\tilde{w}} & =\partial_{s} \tilde{w}-\tilde{\Phi} \Psi(0) g_{0}\left(\tilde{\Phi}(0) v+\tilde{w}_{0}, \tilde{\Phi}(-1) v+\tilde{w}(-1), \tilde{\Phi}\left(-\frac{7}{8}\right) v+\tilde{w}\left(-\frac{7}{8}\right)\right),
\end{aligned}
$$

where $\tilde{w}_{0}=\tilde{w}(0)$ and:

$$
\Psi(0)=\left[\begin{array}{cc}
\frac{169}{300} & -\frac{222179}{144000} \\
\frac{8}{5} & \frac{169}{300} \\
0 & \frac{8}{5}
\end{array}\right], \tilde{\Phi}(s)=\left[\begin{array}{ccc}
1 & s & 1+\frac{s^{2}}{2} \\
0 & 1 & s
\end{array}\right] .
$$

By using the Center Manifold Theorem presented in the previous section and the following changes of coordinates:

$$
\begin{aligned}
a & =-7-\frac{35}{128} \alpha r^{6}, b=8+\frac{5}{16} \gamma r^{2}, \tau_{1}=1-\frac{7}{768} \beta r^{4}, \\
v_{l} & =r^{3} u_{1}, v_{2}=r^{5} u_{2}, v_{3}=r^{7} u_{3}, w=r^{3} q,
\end{aligned}
$$

where $r$ is a sufficiently small parameter, we arrive to the expansion of the graph (the center manifold) in power of $r$ which is of order 6, i.e., $q(u, \mu, r)=r^{6} q_{6}(u, \mu, r)$, where $\mu=(\alpha, \beta, \gamma)$ and the expression of the flow on the local center manifold is

$$
\dot{u}=\left[\begin{array}{lll}
0 & 1 & 0 \\
0 & 0 & 1 \\
\alpha & \beta & \gamma
\end{array}\right] u+\left[\begin{array}{c}
0 \\
0 \\
u_{1}^{3}
\end{array}\right]+r^{2} R(u, \mu, r),
$$

where the remainder is a smooth function $R: \mathbb{R}^{3} \times \mathbb{R}^{3} \times \mathbb{R} \rightarrow \mathbb{R}^{3}$. 
Remark 4. We point out that a larger multiplicity for the zero singularity might occur when using a controller consisting in three delay blocks $D=\sum_{k=1}^{3} a_{k} \theta\left(t-\tau_{k}\right)$, this is a natural consequence of Proposition $2.4^{1}$, see also [6] for further details. Indeed, the zero singularity codimension bound is equal to 4 inducing richer dynamics. Such a configuration is guaranteed by the following values of coefficients:

$$
\left\{\begin{array}{l}
a_{1}=\frac{7}{8} \frac{8 \tau_{2}^{2}+7}{\left(8 \tau_{2} \tau_{1}^{2}-14 \tau_{1}-7 \tau_{2}\right)\left(-\tau_{2}+\tau_{1}\right)}, a_{2}=-\frac{7}{8} \frac{8 \tau_{1}^{2}+7}{\left(-7 \tau_{1}+8 \tau_{1} \tau_{2}^{2}-14 \tau_{2}\right)\left(-\tau_{2}+\tau_{1}\right)}, \\
a_{3}=\frac{\left(64 \tau_{1}^{2} \tau_{2}^{2}-112 \tau_{1} \tau_{2}+49\right)\left(8 \tau_{1} \tau_{2}-7\right)}{8\left(8 \tau_{2} \tau_{1}^{2}-14 \tau_{1}-7 \tau_{2}\right)\left(-7 \tau_{1}+8 \tau_{1} \tau_{2}^{2}-14 \tau_{2}\right)}, \tau_{3}=7 \frac{\tau_{2}+\tau_{1}}{8 \tau_{1} \tau_{2}-7}, \\
\text { where } \tau_{1} \neq \tau_{2}, \quad \tau_{1} \neq \frac{14 \tau_{2}}{-7+8 \tau_{2}^{2}}, \quad \tau_{2} \neq \frac{14 \tau_{2}}{-7+8 \tau_{1}^{2}} \quad \text { and } 8 \tau_{1} \tau_{2}>7 .
\end{array}\right.
$$

\subsection{Concluding remark}

It is important to recall that, in a neighborhood of the origin, the stability of the solution of the normal form (20) in the center manifold proves the local stability of the solution of the initial infinite dimensional system (11). Moreover, one can easily establish values for $\alpha, \beta$ and $\gamma$ so that the matrix associated with the linear part of the normal form (20) be Hurwitz (all eigenvalues with negative real part). Thus, choosing a sufficiently small value for the scale parameter $r$ and using (18) allow us to establish the values of the gains and the delays guaranteeing the stability of the inverted pendulum.

It is important to note that the global bifurcation diagram is beyond the scope of this paper. Furthermore, it is clear from the literature that the bifurcation diagram for the triple zero singularity (in all generality) is very complicated not only from a theoretical point of view but also from a numerical point of view $[9,10,13]$. However, under the $\mathbb{Z}_{2}$ symmetry as for the cubic truncation of (20), we refer the reader to [3] where such a type of symmetry is identified for the Chua equations. The global analysis established in [32] applies for the present configuration since the same cubic truncated normal form is considered.

\section{Notes and comments}

The use of multiple delay blocks was suggested in $[27,17]$ for stabilizing chains of integrators. In this paper, we design such a multi-delayed-proportional controller allowing to stabilize the inverted pendulum by avoiding a triple zero eigenvalue singularity. This singularity was already identified in [32] through the use of a delayed PD controller. Such a singularity underlines an interesting observation: the multiplicity of the zero spectral value might exceed the dimension of the control-free system [6]. We have shown that a multi-delayed-proportional controller allows to offset the derivative gain while keeping the same performance. These results agree with the claim of [4], that is, the effect of the delay is similar to derivative feedback in modifying the behavior of the system. However, we extend the claim to the nonlinear analysis by proving that the cubic truncated normal form of the center manifold dynamics is the same as the one obtained by using a delayed PD regulator. Thus, the global analysis for the codimension- 3 triple zero bifurcation established in [32] applies for the presented configuration.

${ }^{1}$ It is obvious that such a controller implies an additional coefficient 


\section{Acknowledgement}

The authors would like to thank the anonymous reviewers as well as the associate editor for their valuable comments and suggestions to improve the quality of the paper.

\section{References}

[1] L. V. Ahlfors. Complex Analysis. McGraw-Hill, Inc., 1979.

[2] M. Ait Babram, O. Arino, and M. L. Hbid. Computational scheme of a center manifold for neutral functional differential equations. J. Math. Anal. Appl., 258(2):396-414, 2001.

[3] A. Algaba, M. Merino, E. Freire, E. Gamero, and A. J. Rodrigues-Luis. Some results on chua's equation near a triple-zero linear degeneracy. International Journal of Bifurcation and Chaos, 13(03):583-608, 2003.

[4] F. M. Atay. Balancing the inverted pendulum using position feedback. Appl. Math. Lett., 12(5):51-56, 1999.

[5] R. Bellmanand and K. L. Cooke. Differential-difference equations. Academic Press, New York, 1963.

[6] I. Boussaada and S-I. Niculescu. Computing the codimension of the singularity at the origin for delay systems: The missing link with birkhoff incidence matrices. 21st International Symposium on Mathematical Theory of Networks and Systems July 7-11, 2014, Groningen, The Netherlands, pages 1-6, 2014.

[7] S.A. Campbell. Calculating centre manifolds for delay differential equations using maple. In In Delay Differential Equations: Recent Advances and New Directions. Springer-Verlag, 2009.

[8] O. Diekmann, S.A. Van Gils, S.M. Verduyn Lunel, and H.O. Walther. Delay equations, volume 110 of Applied Mathematical Sciences, Functional, complex, and nonlinear analysis. Springer-Verlag, New York, 1995.

[9] F. Dumortier and S. Ibanez. Nilpotent singularities in generic 4-parameter families of 3-dimensional vector fields. Journal of Differential Equations, 127(2):590 - 647, 1996.

[10] F. Dumortier, S. Ibanez, and H. Kokubu. New aspects in the unfolding of the nilpotent singularity of codimension three. Dynamical Systems, 16(1):63 - 95, 2001.

[11] J. Eker and K.J. Aström. A nonlinear observer for the inverted pendulum. The 8th IEEE Conference on Control Application, Dearborn, USA, September 15-18, page 7pp, 1996.

[12] T. Faria and L.T. Magalhães. Normal forms for retarded functional-differential equations with parameters and applications to Hopf bifurcation. J. Differential Equations, 122(2):181-200, 1995.

[13] E. Freire, E. Gamero, A. J. Rodrigues-Luis, and A. Algaba. A note on the triple-zero linear degeneracy: Normal forms, dynamical and bifurcation behaviors of an unfolding. International Journal of Bifurcation and Chaos, 12(12):2799-2820, 2002.

[14] K. Gu, S.-I. Niculescu, and J. Chen. On stability of crossing curves for general systems with two delays. J. Math. Anal. Appl., 311:231-253, 2005.

[15] J. K. Hale and S. M. Verduyn Lunel. Introduction to functional differential equations, volume 99 of Applied Mathematics Sciences. Springer Verlag, New York, 1993.

[16] T. Insperger and G. Stepan. Semi-Discretization for Time-Delay Systems: Stability and Engineering Applications. Applied Mathematics Sciences. Springer, 2011.

[17] V.L. Kharitonov, S-I. Niculescu, J. Moreno, and W. Michiels. Static output feedback stabilization: necessary conditions for multiple delay controllers. IEEE Trans. on Aut. Cont., 50(1):82-86, 2005.

[18] Y. Kuznetsov. Elements of applied bifurcation theory; Second edition, volume 112 of Applied Mathematics Sciences. Springer, New York, 1998.

[19] M. Landry, S.A. Campbell, K. Morris, and C. O. Aguilar. Dynamics of an inverted pendulum with delayed feedback control. SIAM J. Appl. Dyn. Syst., 4(2):333-351 (electronic), 2005.

[20] C. Mendez-Barrios, S.-I. Niculescu, C.-I. Morarescu, and Keqin Gu. On the fragility of pi controllers for time-delay siso systems. In Control and Automation, 2008 16th Mediterranean Conference on, pages 529-534, June 2008.

[21] W. Michiels and S-I. Niculescu. Stability and stabilization of time-delay systems, volume 12 of Advances in Design and Control. Society for Industrial and Applied Mathematics (SIAM), Philadelphia, PA, 2007.

[22] J. Milton, J-L. Cabrera, T. Ohira, S. Tajima, Y. Tonosaki, C.W. Eurich, and Sue Ann Campbell. The time-delayed inverted pendulum: Implications for human balance control. Chaos, (19):12pp, 2009.

[23] I.-C. Morărescu, W. Michiels, and M. Jungers. Synchronization of coupled nonlinear oscillators with gammadistributed delays. In Proceedings of IEEE American Control Conference, 2013.

[24] I.-C. Morărescu, S.-I. Niculescu, and K.Gu. On the geometry of stability regions of smith predictors subject to delay uncertainty. IMA Journal of Mathematical Control and Information, 24(3):411-423, 2007.

[25] I.-C. Morărescu, S.-I. Niculescu, and K.Gu. Stability crossing curves of shifted gamma-distributed delay systems. SIAM Journal on Applied Dynamical Systems, 6(2):475-493, 2007.

[26] N. Muskinja and B. Tovornik. Swinging up and stabilisation of a real inverted pendulum. IEEE Transaction on Industrial Electronics, 53(2):631-639, 2006. 
[27] S-I. Niculescu and W. Michiels. Stabilizing a chain of integrators using multiple delays. IEEE Trans. on Aut. Cont., 49(5):802-807, 2004.

[28] N. Olgac and R. Sipahi. An exact method for the stability analysis of time delayed linear time-invariant (lti) systems. IEEE Transactions on Automatic Control, 47(5):793-797, 2002.

[29] G. Polya and G. Szegö. Problems and Theorems in Analysis, Vol. I: Series, Integral Calculus, Theory of Functions. Springer-Verlag, New York, Heidelberg, and Berlin, 1972.

[30] A. Seuret, C. Edwards, S.K. Spurgeon, and E. Fridman. Static output feedback sliding mode control design via an artificial stabilizing delay. Automatic Control, IEEE Transactions on, 54(2):256-265, Feb 2009.

[31] A. Shiriaev, A. Pogromsky, H. Ludvigsen, and O. Egeland. On global properties of passivity-based control of an inverted pendulum. Int. J. of Robust Nonlinear Control, 10:283-300, 2000.

[32] J. Sieber and B. Krauskopf. Bifurcation analysis of an inverted pendulum with delayed feedback control near a triple-zero eigenvalue singularity. Nonlinearity, 17:85-103, 2004

[33] J. Sieber and B. Krauskopf. Extending the permissible control loop latency for the controlled inverted pendulum. Dynamical Systems, 20(2):189-199, 2005.

[34] R. Sipahi and N. Olgac. Complete robustness of third-order lti multiple time-delay systems. Automatica, 41:14131422, 2005.

[35] I.H. Suh and Z. Bien. Proportional minus delay controller. IEEE Trans. on Aut. Cont., AC24:370-372, 1979. 